\title{
SURVIVAL AND FUNCTION AFTER SLEEVE LOBECTOMY FOR LUNG CANCER
}

Henning A. Gaissert, $\mathrm{MD}^{\S}$

Douglas J. Mathisen, MD

Ashby C. Moncure, MD

Alan D. Hilgenberg, MD

Hermes C. Grillo, MD

John C. Wain, $\mathrm{MD}^{\S}$
Between 1962 and 1991, 72 patients (mean age 63.4 years) underwent sleeve lobectomy for primary lung cancer. Thirty-seven patients had adequate lung function and 35 were deemed unsuitable for pneumonectomy on the basis of inadequate pulmonary reserve $(n=31)$ or cardiac risk factors $(n=$ 4). Squamous cell carcinomas $(68 \%)$ and adenocarcinomas $(26 \%)$ predominated. Upper lobectomy was performed in 48 patients, lower and middle lobectomy in 13, and right upper and middle bilobectomy in 11. Hospital mortality was $4 \%(3 / 72)$ and compares with a hospital mortality of $9 \%$ in 56 consecutive pneumonectomies between 1986 and 1990. Major complications occurred in 11\% (bronchopleural fistula 1, persistent atelectasis 4, pneumonia 4). Adjusted actuarial survival after sleeve lobectomy at 1 and 5 years was $84 \%$ and $42 \%$, compared with $76 \%$ and $44 \%$ after pneumonectomy. Five-year survival after lower and middle lobectomy in 13 patients (52\%) was similar to that after upper lobectomy (46\%), suggesting that in carefully selected patients the concept of sleeve lobectomy can be applied to all pulmonary lobes. $\mathrm{N} 1$ disease and compromised lung function were associated with lower survival (N1 38\% vs N0 57\%; compromised $20 \%$ vs adequate $55 \%$ ). Comparison of preoperative and postoperative lung function and quantitative ventilation-perfusion isotope studies substantiated the preservation of pulmonary function in this group of patients. Sleeve lobectomy is the procedure of choice for anatomically suitable carcinomas or when reduced pulmonary reserve precludes extensive resection. (J Thorac Cardiovasc Surg 1996;111:948-53)
$S^{1}$ eeve resection of the bronchus has changed the management of benign tumors ${ }^{1}$ and altered the approach to selected malignant bronchogenic neoplasms. ${ }^{2,3}$ Bronchoplastic procedures for lung cancer were originally designed for patients with compromised pulmonary function unable to tolerate pneumonectomy. As it became likely that sleeve lobectomy yielded survival results at least equal to those of pneumonectomy, and yielded better functional results, it became an accepted procedure for

From the General Thoracic Surgical Unit, Massachusetts General Hospital, Harvard Medical School, Boston, Mass.

Read at the Seventy-third Annual Meeting of The American Association for Thoracic Surgery, Chicago, Ill., April 25-28, 1993.

Received for publication Sept. 20, 1995; revisions requested Oct. 19, 1995; revisions received Dec. 11, 1995; accepted for publication Dec. 13, 1995

Address for reprints: Douglas J. Mathisen, MD, Warren 1109, Massachusetts General Hospital, Boston, MA 02114.

${ }^{\S}$ By invitation.

Copyright (C) 1996 by Mosby-Year Book, Inc.

$0022-5223 / 96 \$ 5.00+0 \quad \mathbf{1 2 / 6 / 7 1 1 6 6}$ patients with lung cancer who have anatomically suitable tumors, regardless of pulmonary function.

Limited information is available regarding longterm functional results and survival despite the growing experience with sleeve lobectomy. Early postoperative lung dysfunction after sleeve lobectomy has been demonstrated in animals and human beings ${ }^{4,5}$ but does not appear to affect long-term performance. It has been assumed that preserved lung functions normally, but little information exists to confirm this assumption.

The reported 5-year survival for sleeve lobectomy ranges from $18 \%$ to $67 \%$, depending on stage and pulmonary functional reserve. ${ }^{6-9}$ Patients with normal lung function achieve better long-term survival than those with compromised function. No randomized trials comparing sleeve lobectomy with pneumonectomy have been performed, and they are not likely to be performed. Thus the available information is derived from unmatched, retrospective cohorts of patients.

We have reviewed our experience with sleeve lobectomy in a consecutive series of 72 patients to 
verify or refute results obtained by others. We determined perioperative morbidity and mortality, long-term survival, and late functional results. Because pneumonectomy is the surgical alternative to sleeve lobectomy, we chose a recent 5-year interval to compare morbidity, mortality, and survival when appropriate in a consecutive, unmatched group of patients undergoing pneumonectomy for lung cancer.

\section{Patients and methods}

Seventy-two patients underwent sleeve lobectomy for bronchogenic carcinoma at the Massachusetts General Hospital from 1962 to 1991. Resections involving the carina were excluded. Information was gathered from hospital charts, office records, and personal contact with patients and their families. Inasmuch as the surgical alternative to sleeve lobectomy is pneumonectomy, a consecutive, unmatched series of 56 patients undergoing pneumonectomy for bronchogenic carcinoma between 1986 and 1990 at the same institution provided some comparison of perioperative morbidity, mortality, and long-term survival; the comparison is somewhat prejudicial to sleeve lobectomy, in view of the recency of the pneumonectomy operations. Although no attempt was made to match the groups, they were fairly comparable in terms of age, sex, histology, stage, and nodal status (Table I).

Statistical methods. Cumulative survival was calculated according to the actuarial method of Cutler and Ederer modified by Anderson and associates. ${ }^{10}$

Preoperative evaluation. Computed axial tomograms of the chest, upper part of the abdomen, and adrenal glands, or linear tomograms in those operated on before 1976, were obtained in all patients. Metastatic workup including head computed tomography and bone scan was performed when indicated. Preoperative pulmonary function studies were done in all patients to determine firstsecond forced expiratory volume $\left(\mathrm{FEV}_{1}\right)$ and forced vital capacity. Quantitative ventilation and perfusion radionuclide scans were performed in 50 patients $(69 \%)$. Postoperative $\mathrm{FEV}_{1}$ was predicted by the formula: postoperative $\mathrm{FEV}_{1}=$ preoperative $\mathrm{FEV}_{1} \times$ percent function of lung regions not to be resected. ${ }^{11} \mathrm{~A}$ predicted postoperative $\mathrm{FEV}_{1}$ of less than $800 \mathrm{ml} / \mathrm{sec}$ was considered a contraindication for pneumonectomy. On the basis of clinical and objective functional assessment, 37 patients had adequate pulmonary function to tolerate pneumonectomy and 35 patients were not in suitable condition for pneumonectomy. Twenty-five had inadequate lung function at spirometry to tolerate pneumonectomy, seven had clinical signs of inadequate lung function, two had cardiovascular contraindications, one was elderly, and one had multiple sclerosis.

Surgical technique. The procedure for sleeve lobectomy is identical to that for standard lobectomy until the bronchus is isolated. A single sharp incision divides the bronchus and thus avoids ragged edges. Care is taken not to devascularize the bronchus beyond the proposed line of transection. We refrain from any handling of the bron-
Table I. Patient characteristics*

\begin{tabular}{|c|c|c|c|c|}
\hline & \multicolumn{2}{|c|}{$\begin{array}{c}\text { Sleeve } \\
\text { lobectomy } \\
(n=72) \text { : } \\
1962-1991\end{array}$} & \multicolumn{2}{|c|}{$\begin{array}{l}\text { Pneumonectomy } \\
\quad(n=56): \\
1986-1990\end{array}$} \\
\hline & No. & $\%$ & No. & $\%$ \\
\hline Men & 56 & 78 & 42 & 75 \\
\hline Women & 16 & 22 & 14 & 25 \\
\hline \multicolumn{5}{|l|}{ Histology } \\
\hline Squamous & 49 & 68 & 33 & 59 \\
\hline Adenocarcinoma & 19 & 26 & 17 & 30 \\
\hline Large cell & 3 & 4.2 & 2 & 3.5 \\
\hline Adenosquamous & 1 & 1.4 & 2 & 3.5 \\
\hline Bronchoalveolar & & & 2 & 3.5 \\
\hline \multicolumn{5}{|l|}{ Stage } \\
\hline I & 29 & 40 & 9 & 16 \\
\hline II & 31 & 43 & 25 & 45 \\
\hline III & 12 & 17 & 21 & 37 \\
\hline IV & & & 1 & 2 \\
\hline \multicolumn{5}{|l|}{ Nodal stage } \\
\hline No & 31 & 43 & 15 & 26 \\
\hline N1 & 34 & 47 & 32 & 57 \\
\hline $\mathrm{N} 2$ & 7 & 10 & 9 & 17 \\
\hline
\end{tabular}

* Mean age for the sleeve lobectomy group was 63.4 years (range 35 to 86 years) and for the pneumonectomy group, 60.8 years (range 37 to 77 years).

chial mucosa. Frozen section analysis is usually performed by sending a thin ring of tissue from the two ends of the airway to be anastomosed. No attempt is made to tailor either end of the bronchus. Traction sutures of 2-0 Vicryl polyglactin 910 (Ethicon, Inc., Somerville, N.J.) are placed proximal and distal in the mid-lateral position of the bronchial wall. Individual sutures of 4-0 Vicryl polyglactin 910 are evenly placed. Proper spacing of the sutures will accommodate any size discrepancy between the two ends of the airway. After approximation of the traction sutures, the sutures are tied circumferentially with the knots on the outside. The anastomosis is checked for air leaks by inflating the lung after filling the thorax with saline solution. The suture line is inspected with a flexible bronchoscope at this point. Pedicled pleura or pericardial fat is used to wrap the bronchus to separate the suture line from vascular structures.

\section{Results}

Age and sex. The sleeve lobectomy group consisted of 56 men and 16 women with a mean age of 63.4 years (range 35 to 86 years). The pneumonectomy group comprised 42 men and 14 women. Their ages ranged from 37 to 77 years with a mean of 60.8 years.

Staging and histology. Histologic characteristics for both groups are seen in Table I. Sixty-one patients had mediastinoscopy and three patients had a Chamberlain procedure before sleeve lobectomy. All but three of the patients had disease-free medi- 


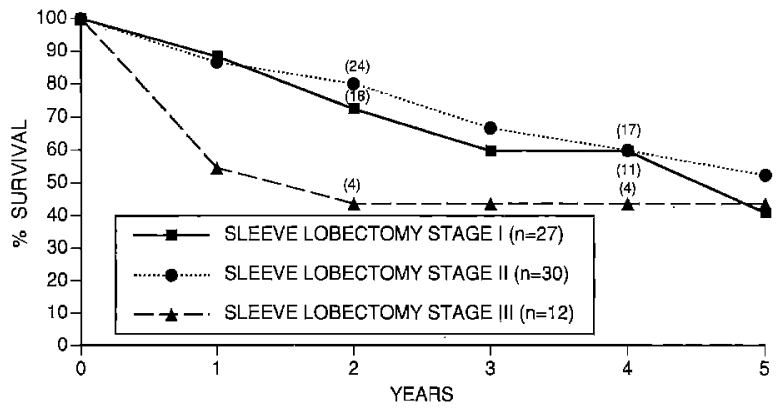

Fig. 1. Actuarial survival after sleeve lobectomy by stage.

astinal nodes. The three patients with diseased N2 nodes were entered into a neoadjuvant treatment protocol. In the pneumonectomy group, 42 patients underwent mediastinoscopy and six a Chamberlain procedure. Postoperative pathologic staging for both groups is given in Table I.

Indication for sleeve lobectomy. The presence of an endobronchial tumor identified during bronchoscopy precluded standard lobectomy in 54 patients. In the other 18 patients, the decision for sleeve lobectomy was made during thoracotomy. Metastatic lymph nodes adherent to the origin of the lobar bronchus were found in eight patients. Direct extension from central tumors onto the wall of the bronchus not detected by bronchoscopic study was seen in six patients. Unsuspected involvement of the bronchial margins was diagnosed by frozen section in four patients and necessitated sleeve lobectomy.

Type of resection. Right upper sleeve lobectomy was performed in 38 patients (53\%), sleeve lobectomy of right upper and middle lobes in $11(15 \%)$, left upper lobe in $10(14 \%)$, left lower lobe in six $(8 \%)$, right middle lobe in three $(4 \%)$, and right middle and lower lobes in three $(4 \%)$, and right lower lobe in one. When the middle and right upper lobes were resected together, the middle lobe bronchus was taken separately and not included in the bronchial sleeve ${ }^{8}$ in eight patients, and it was taken with the sleeve in three. Concomitant chest wall resection was done in three patients. Sleeve resection of the pulmonary artery was performed in two patients with compromised lung function and lateral resection of the artery in one other patient.

Mortality and morbidity. Three deaths occurred after sleeve lobectomy, resulting in a hospital mortality of $4 \%$. Causes of death were ventricular fibrillation on day 2, myocardial infarction on day 8 in a patient with concomitant sleeve resection of the pulmonary artery, and Pseudomonas pneumonia on
Table II. Morbidity

\begin{tabular}{lcc}
\hline \multicolumn{1}{c}{ Major complications } & $\begin{array}{c}\text { Sleeve lobectomy } \\
(n=72)\end{array}$ & $\begin{array}{c}\text { Pneumonectomy } \\
(n=56)\end{array}$ \\
\hline Bronchpleural fistula & 1 & 1 \\
Pneumonia & 3 & 2 \\
Cerebrovascular accident & & 1 \\
Pulmonary embolus & & 1 \\
Deep vein thrombosis & & 1 \\
Herniation of heart & & 1 \\
Persistent atelectasis & 4 & 1 \\
Total & $8[11 \%]$ & $8[14 \%]$ \\
\hline
\end{tabular}

day 33. No hospital deaths have occurred in 42 consecutive patients since 1986, and no deaths have occurred during the entire series in patients with adequate pulmonary function.

Sleeve lobectomy. Major complications occurred in eight patients (11\%). One patient had a bronchopleural fistula, which necessitated operative repair 2 weeks after lobectomy and was followed by an empyema and prolonged mechanical ventilation. Three patients had pneumonia and four had persistent atelectasis necessitating repeated bronchoscopic treatment for bronchial toilet. Supraventricular tachycardias occurred in nine patients.

Late bronchial stenosis. Bronchial stenosis at the site of the anastomosis developed in two patients. One underwent pneumonectomy 1 year later for retained secretions and recurrent pneumonia. The other patient required several dilatations and has a stable narrowing of the anastomosis with normally functioning lung on the side of the operation 6 years later.

Pneumonectomy. The hospital mortality in 56 patients undergoing pneumonectomy for bronchogenic carcinoma was $9 \%$. The cause of death in five patients was adult respiratory distress syndrome $(n=2)$, bronchopleural fistula $(n=1)$, pneumonia $(n=1)$, and myocardial infarction $(n=1)$. Major complications occurred in 16\% (9/56), consisting of pulmonary embolus $(n=2)$, pneumonia $(n=2)$, cardiac herniation after pericardial resection $(n=$ $1)$, bronchpleural fistula $(n=1)$, stroke $(n=1)$, deep vein thrombosis $(n=1)$, and atelectasis $(n=$ 1 ). Seven patients had supraventricular tachycardia.

Radiotherapy. Radiotherapy was administered on the basis of the surgeon's judgment, most often for hilar lymph node involvement. Twenty-seven patients received postoperative radiation. The indication in 19 was $\mathrm{N} 1$ disease and in two N2 disease. The remaining six patients received postoperative radiotherapy for diseased bronchial margins on perma- 


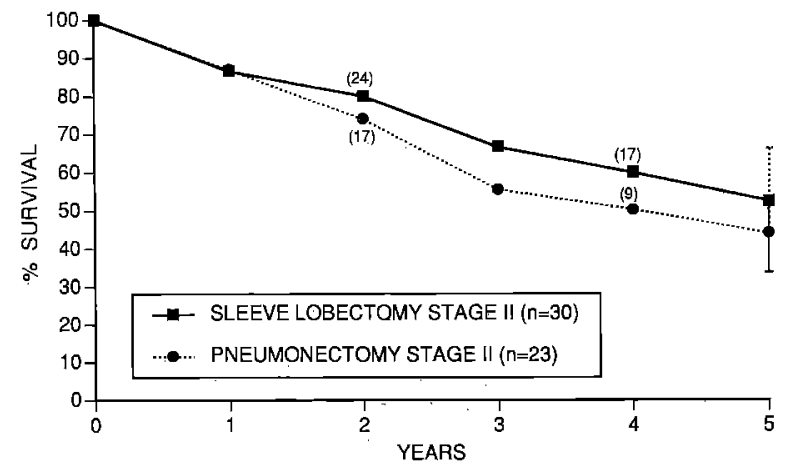

Fig. 2. Comparison of survival after sleeve lobectomy and pneumonectomy in patients with stage II disease. Error bars show $95 \%$ confidence limits.

nent sections ( $n=2)$, carcinoma-in-situ at the bronchial margin $(n=2)$, and diseased venous resection margins $(n=2)$. Three patients early in the series had preoperative radiation, and three with $\mathrm{N} 2$ disease underwent preoperative and postoperative radiation therapy as part of a multimodality protocol.

Follow-up and survival. Follow-up was complete except for one patient in each group. Mean follow-up was $47 \pm 46$ months (mean \pm standard deviation, range 3 to 268 months) after sleeve lobectomy and $37 \pm 25$ months (range 2 to 80 months) after pneumonectomy. Included in survival calculations were two patients who underwent pulmonary resection for a second primary lung cancer 9 and 12 months after sleeve lobectomy.

Actuarial survival by stages in the sleeve lobectomy group is given in Fig. 1. Actuarial survival after sleeve lobectomy was $80 \% \pm 4.8 \%$ (mean \pm standard error) at 1 year and $42 \% \pm 6.5 \%$ at 5 years. These figures compare with actuarial survivals after pneumonectomy of $76 \% \pm 5.9 \%$ at 1 year and $44 \% \pm 7.3 \%$ at 5 years. Fig. 2 shows that the survival in patients with stage II disease after sleeve lobectomy and pneumonectomy was comparable. Survival data were not calculated for pneumonectomy patients with stage I and III disease because of the small numbers. Fig. 3 compares the survival of patients whose functional status was compromised and that of patients whose status was adequate.

Comparison of survival between 47 patients undergoing sleeve resection of the upper lobe (adequate function, 24 patients; compromised function, 23 patients) and 13 patients with middle and lower sleeve lobectomies (adequate, 7 patients; compromised, 5 patients) is demonstrated in Fig. 4. Survival at 5 years was $46 \% \pm 8.5 \%$ after

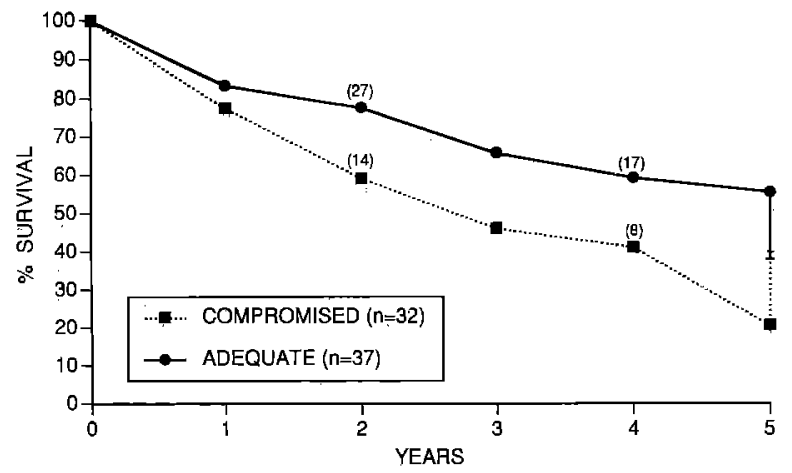

Fig. 3. Actuarial survival in patients with compromised and adequate lung function. Error bars show 95\% confidence limits.

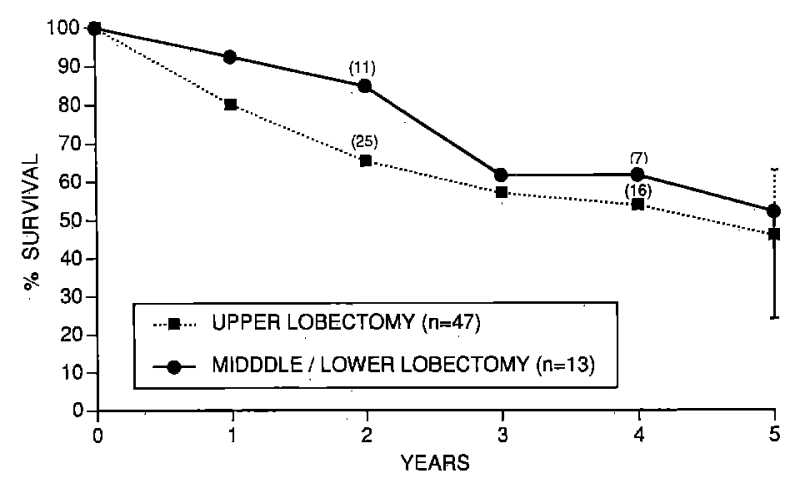

Fig. 4. Actuarial survival in patients after upper versus middle and lower lobectomy. Error bars show 95\% confidence limits.

upper lobectomy and $52 \% \pm 14 \%$ after middle and lower lobectomy. Thirty-one patients with N0 disease had 1 - and 5-year adjusted survivals of $93 \% \pm 4.6 \%$ and $57 \% \pm 10 \%$. Survival in 33 patients with N1 disease was $79 \% \pm 7.1 \%$ and $38 \% \pm 9.3 \%$ and in seven patients with $\mathrm{N} 2$ disease, $57 \% \pm 19 \%$ and $43 \% \pm 19 \%$, respectively. Thirty-six patients not requiring radiation therapy had $91 \% \pm 4.9 \%$ survival at 1 year and $58 \% \pm 9.4 \%$ at 5 years, compared with $69 \%$ $\pm 8.0 \%$ and $30 \% \pm 8.8 \%$ in 33 patients undergoing radiotherapy. The patients receiving radiotherapy could be expected to have a worse prognosis because the indication for this therapy was diseased nodes or margins.

Local recurrence. Locoregional recurrence developed in 10 patients after sleeve lobectomy (14\%; $10 / 69)$. Tumor recurred in the mediastinum in seven patients and in the ipsilateral lung in two; one patient had a confirmed suture-line recurrence. 


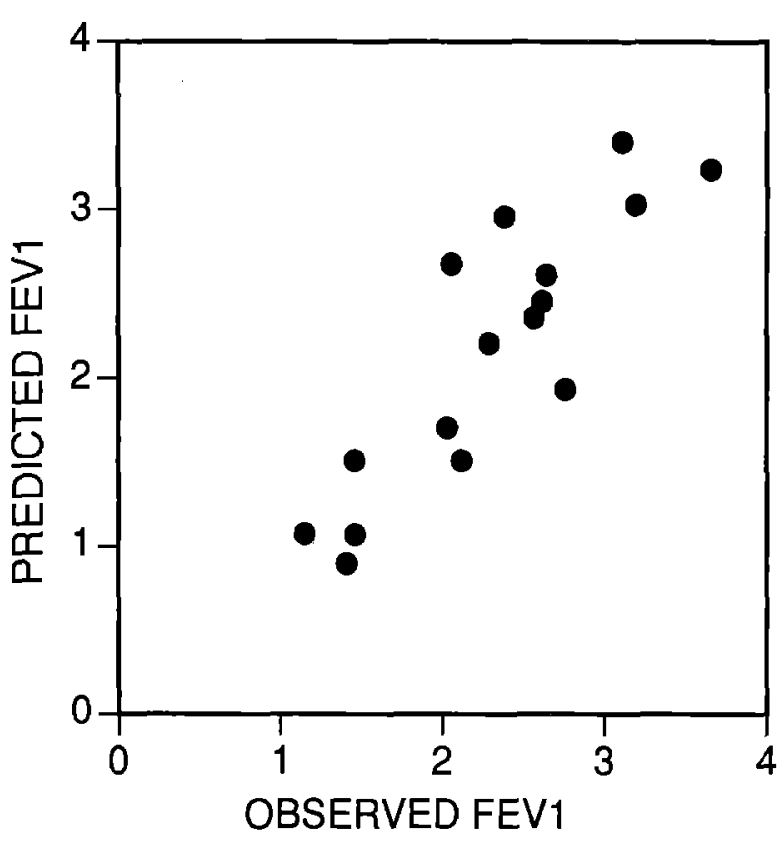

Fig. 5. Distribution of predicted and observed $\mathrm{FEV}_{1}$ in 16 patients. Predicted $\mathrm{FEV}_{1}$ was derived from preoperative pulmonary function tests and quantitative ventilationperfusion scan. ${ }^{11}$ Correlation coefficient is 0.87 .

Prediction of postoperative $F E V_{1}$. In 16 patients with sleeve lobectomy, preoperative and postoperative pulmonary function studies and preoperative quantitative ventilation-perfusion scans were available for comparison. Postoperative pulmonary function studies were obtained 4 months to 11 years (mean 3.5 years) after resection, and eight patients had received radiotherapy. Comparison of predicted and observed $F E V_{1}$, shown in Fig. 5, demonstrates that perfusion-derived data served as a good estimation of postoperative function, with a correlation coefficient of 0.87 . Predicted values overestimated observed values on average by $15 \%$.

Ventilation-perfusion scans. Eight patients had preoperative and postoperative quantitative ventilation-perfusion lung scans 1 to 11 years (mean 4.5 years) after sleeve lobectomy (right upper lobe, 5; left upper lobe, 1; right upper and middle lobes, 1; left lower lobe, 1). Three had undergone postoperative radiotherapy. On the side of the operation, mean postoperative perfusion ratio was $35 \%$ (range $21 \%$ to $48 \%$ ). The lowest perfusion values were observed in a patient with postoperative bronchial stenosis $(21 \%)$ and after radiation therapy $(22 \%)$. Mean postoperative ventilation ratio was $44 \%$
Table III

\begin{tabular}{lllll} 
& & & & \\
& & Predicted & \multicolumn{1}{c}{ Observed } \\
& Preoperative & postoperative & postoperative \\
\hline $\mathrm{FEV}_{1}(\mathrm{~L} / \mathrm{min})$ & $3.28(2.43-4.04)$ & $2.80(2.2-3.4)$ & $2.74(2.06-3.66)$ \\
Perfusion (\%) & $44.5(35-48)$ & $31.4(24-39)$ & $35.0(21-48)$ \\
Ventilation (\%) & $46.2(38-54)$ & $31.7(22-39)$ & $46.2(32-56)$ \\
\hline
\end{tabular}

Comparison of preoperative to predicted and observed postoperative parameters of pulmonary function in eight patients on the operated side. Numbers represent mean values and their range.

(range $32 \%$ to $51 \%$ ). Table III compares preoperative to predicted and observed postoperative $F E V_{1}$ and ventilation/perfusion ratios on the side of the operation.

\section{Discussion}

Sleeve lobectomy for anatomically suited lung cancers has become the procedure of choice for patients with compromised and noncompromised lung function. Before the development of segmental bronchial resection, cancers of the origin of the lobar bronchus necessitated pneumonectomy to remove all of the cancer. No prospective studies comparing sleeve lobectomy and pneumonectomy have been conducted. Comparable mortality and survival for both procedures have been demonstrated in retrospective studies. ${ }^{6,7}$ In our series, the entire group of patients having sleeve lobectomies had an operative mortality of $4 \%$, compared with $9 \%$ for the pneumonectomy group. No deaths related to the operation occurred among patients with adequate function undergoing sleeve resection. Major complications were more common after pneumonectomy (16\%) than after sleeve lobectomy $(11 \%)$. Although the comparison of these two groups was retrospective and nonrandomized, these data suggest the superiority of sleeve lobectomy over pneumonectomy in selected patients.

The overall survival after sleeve lobectomy is virtually identical to that after pneumonectomy in our series. Analysis of subsets within the sleeve lobectomy group indicates improved survival for patients with noncompromised lung function compared with compromised function (55\% vs $20 \%$ 5-year survival) and for patients with N0 compared with N1 disease ( $57 \%$ vs $38 \%$ at 5 years) ${ }^{12}$ The difference in survival between patients who did or did not receive radiation therapy reflects nodal status, because the indication for radiation therapy was predominantly hilar or mediastinal nodal involvement. Comparison of survival between sleeve lobectomy and pneumonectomy according to nodal 
Volume 111, Number 5

status and stage demonstrated little difference between the two procedures (see Fig. 2).

Limited information is available on the outcome after sleeve resection of the middle and lower lobes. Weisel and associates ${ }^{6}$ reported eight lower lobectomies in 70 patients and Firmin and colleagues ${ }^{8}$ reported four lower lobe resections among 90 patients. Middle and lower sleeve lobectomies are done less often because suitable intrabronchial lesions are rare. Furthermore, on the basis of studies on the lymphatic spread of lung cancer, some authors believe that a bronchoplastic procedure does not adequately encompass central tumors of the lower and middle lobes and thus results in inferior tumor clearance. In our series the actuarial survival was $52 \%$ for 13 patients with this type of resection compared with $46 \%$ after upper sleeve lobectomy. These data suggest that the concept of parenchyma-preserving resection can be applied to all pulmonary lobes.

Chances for survival appear remote when sleeve resection is performed for carcinomas invading the bronchial wall from the peribronchial tissues and not from the luminal side. This indication was present in six patients without intrabronchial tumor, of whom three had stage I disease, one stage II, and two stage III disease. All except one patient would not have tolerated a more radical procedure on the basis of preoperative lung function. Five of them died 6 to 22 months after operation of recurrent tumor and one was lost early to follow-up. Careful consideration of pneumonectomy must be given to this group of patients if lung function permits.

With survival equal to or greater than after pneumonectomy, the principal benefit of sleeve lobectomy has been the ability to preserve lung parenchyma. ${ }^{9,13}$ Preoperative assessment predicted postoperative lung function reliably in our patients. Quantitative ventilation and perfusion scans demonstrated that the contribution to postoperative lung function was proportionally distributed between the preserved lung that had been operated on and the contralateral side. Mean postoperative perfusion to the side of the operation was $35 \%$ in these patients after upper or lower lobectomy, indicating that the expected proportion of pulmonary blood flow is directed to the preserved lung.

In patients with adequate lung function, sleeve lobectomy should never compromise the cancer operation. Locoregional recurrence developed in 10 of 69 patients $(14 \%)$. In seven patients, tumors recurred in the mediastinum, where tumor clearance would unlikely be improved by a pneumonec- tomy. Only three patients had a local recurrence in the ipsilateral lung $(n=2)$ or at the suture line $(n=$ 1). Pneumonectomy may offer little additional advantage for close bronchial margins unless carinal resection is considered. Clinical judgment must be exercised to decide between sleeve lobectomy and pneumonectomy in some patients with central perihilar neoplasms.

Sleeve lobectomy is indeed the procedure of choice in patients with lung cancer who have anatomically suited tumors for this procedure. Survival is acceptable and appears comparable with the surgical alternative of pneumonectomy. Improved quality of life can be expected because of the preservation of functioning lung.

\section{REFERENCES}

1. Wilkins EW, Grillo HC, Moncure AC, Scannell JG. Changing times in surgical management of bronchopulmonary carcinoid tumor. Ann Thorac Surg 1964;38:339-44.

2. Paulson DL, Urschel HC, McNamara JJ, Shaw RR. Bronchoplastic procedures for bronchogenic carcinoma. J Thorac Cardiovasc Surg 1970;59:38-47.

3. Jensik RJ, Faber LP, Milloy FJ, Amato JJ. Sleeve lobectomy for carcinoma. J Thorac Cardiovasc Surg 1972;64:400-412.

4. Wood PB, Gilday D, Ilves R, Rae S, Pearson FG. A comparison of gas exchange after simple lobectomy and lobectomy with sleeve resection in dogs. J Thorac Cardiovasc Surg 1974;68:646-53.

5. Brusasco V, Ratto GB, Crimi P, Sacco A, Motta G. Lung function following upper sleeve lobectomy for bronchogenic carcinoma. Scand J Thorac Cardiovasc Surg 1988;22:73-8.

6. Weisel RD, Cooper JD, Delarue NC, Theman TE, Todd TRJ, Pearson FG. Sleeve lobectomy for carcinoma of the lung. J Thorac Cardiovasc Surg 1979;78:839-49.

7. Faber LP, Jensik RJ, Kittle CF. Results of sleeve lobectomy for bronchogenic carcinoma in 101 patients. Ann Thorac Surg 1984;37:279-85.

8. Firmin RK, Azariades M, Lennox SC, Lincoln JCR, Paneth M. Sleeve lobectomy (lobectomy and bronchoplasty) for bronchial carcinoma. Ann Thorac Surg 1983;35:442-9.

9. Deslauriers J, Gaulin P, Beaulieu M, Piraux M, Bernier R, Cormier Y. Long-term clinical and functional results of sleeve lobectomy for primary lung cancer. J Thorac Cardiovasc Surg 1986;92:871-9.

10. Anderson RP, Bonchek LI, Grunkemeier GL, Lambert LE, Starr A. The analysis and presentation of surgical results by actuarial methods. J Surg Res 1974;16:224-30.

11. Bria WF, Kanarek DJ, Kazemi H. Prediction of postoperative pulmonary function following thoracic operations. Value of ventilation-perfusion scanning. J Thorac Cardiovasc Surg 1983;86:186-92

12. Mehran RJ, Deslauriers J, Piraux M, Beaulieu M, Guimont C, Brisson J. Survival related to nodal status after sleeve resection for lung cancer. J Thorac Cardiovasc Surg 1994; 107:576-83.

13. Khargi K, Duurkens VAM, Verzijlbergen FF, Huysmans HA, Knaepen PJ. Pulmonary function after sleeve lobectomy. Ann Thorac Surg 1994;57:1302-4. 